\title{
A CLOSED FORM SOLUTION TO SEMI-BLIND JOINT SYMBOL AND CHANNEL ESTIMATION IN MIMO-OFDM SYSTEMS
}

\author{
Kefei Liu ${ }^{1}$, João Paulo C. L. da Costa ${ }^{2}$, André L. F. de Almeida ${ }^{3}$, and H.C. So ${ }^{1}$ \\ ${ }^{1}$ Department of Electronic Engineering, City University of Hong Kong, Hong Kong \\ ${ }^{2}$ University of Brasília, Electrical Engineering Department, P.O. Box 4386, 70910-900 Brasília, Brazil \\ ${ }^{3}$ GTEL-Wireless Telecom Research Group, Federal University of Ceará, Brazil \\ E-mail:kefeilau@gmail.com,jpdacosta@unb.br,andre@gtel.ufc.br,hcso@ee.cityu.edu.hk
}

\begin{abstract}
Due to the scarcity of the electromagnetic spectrum, multidimensional signaling schemes that take into account several signal dimensions such as space, time, frequency and constellation, are good candidates for increasing the data rate and/or improving the link reliability in future communication systems. Recently a new space-time-frequency diversity based MIMO-OFDM system has been proposed where transmit signal design combines frequency-domain Vandermonde spreading with a time-varying linear constellation precoding, while the received signal is formulated as a nested parallel factor (PARAFAC) model. A joint channel estimation and symbol decoding process has been developed for this system based on the alternating least squares (ALS) algorithm. In this paper, we propose a low-complexity blind receiver based on the least squares Khatri-Rao factorization (LS-KRF) for joint channel estimation and symbol decoding. Our proposed LS-KRF receiver is a closed-form solution which provides the same performance as that of the ALS solution while being less complex since no iteration is needed. Simulation results are included to verify the benefits of the proposed receiver.
\end{abstract}

Index Terms - MIMO-OFDM, nested PARAFAC, least squares Khatri-Rao factorization, blind receiver, linear constellation precoding

\section{INTRODUCTION}

The combination of multiple input multiple output (MIMO) systems and orthogonal frequency division multiplexing (OFDM) has been a focus of a large number of works [1]. In MIMO-OFDM, transmit antennas are employed to achieve high data rates via spatial multiplexing as well as to improve link reliability in frequency-selective channels through space-time/space-frequency or space-time-frequency coding [2-7]. In a number of recent works, space-time codes

\footnotetext{
The work described in this paper was supported by a grant from the Research Grants Council of the Hong Kong Special Administrative Region, China (Project no. CityU 120911), and the work of A. L. F. de Almeida is partially supported by the CNPq/Brazil (Proc. 303238/2010-0).
}

with blind detection have been proposed using tensor decompositions [8-11]. In [9], joint space-time spreading based transmit diversity is considered, and a constrained tensor model is used for blind detection. More general space-time spreading structures are recently proposed relying on a threeway constrained factor (CONFAC) model [10].

Recently, a new space-time-frequency coding structure based on nested parallel factor (PARAFAC) model has been proposed in MIMO-OFDM systems [11]. The proposed transmit signal design combines frequency-domain Vandermonde spreading with a time-varying linear constellation precoding. The received data is formulated as a nested PARAFAC model and then a blind receiver based on the alternating least square (ALS) algorithm is developed for blind symbol and channel estimation. However, such a solution has a complexity that depends on the number of iterations and convergence to the global optimum is not guaranteed due to the sensitivity of ALS to parameter initialization..

The least squares Khatri-Rao factorization (LS-KRF) proposed in [12] for MIMO relaying problems has also been successfully applied for parameter estimation problems in [13]. In this work, we propose a least squares Khatri-Rao factorization (LS-KRF) blind receiver to solve the joint symbol and channel estimation problem in the space-time-frequency MIMO-OFDM system proposed in [11]. This blind receiver exploits the Khatri-Rao structure of the received signal tensor. The key feature of the proposed LS-KRF receiver is its simplicity as compared to the ALS one in terms of the mean processing time, since no iteration is required. Moreover, for some configurations the LS-KRF receiver outperforms the ALS solution in terms of bit error rate.

This paper is organized as follows. In Section 2, the system model is described, where the receive data is formulated as a nested PARAFAC model. This model is exploited for blind symbol decoding and channel estimation in Section 3, where we present the tensor formulation of the received data and describe our proposed LS-KRF receiver. Numerical results are presented in Section 4, and conclusion is drawn in Section 5 . 
Notation: Scalars are denoted by lower-case letters $(a, b, \cdots)$, vectors are written as boldface lower-case letters $(\boldsymbol{a}, \boldsymbol{b}, \cdots)$, matrices as boldface capitals $(\boldsymbol{A}, \boldsymbol{B}, \cdots)$, and tensors as boldface calligraphic letters $\mathcal{A}, \mathcal{B}, \cdots)$. The superscripts ${ }^{T}, H,{ }^{\dagger}$, and ${ }^{*}$ represent transpose, Hermitian transpose, pseudo-inverse and complex conjugate of a matrix, respectively. $\boldsymbol{A}(:, i) \in \mathbb{C}^{R \times 1}$ is a column vector denoting the $i$-th column of $\boldsymbol{A} \in \mathbb{C}^{I \times R}$. The operator $\operatorname{diag}(\boldsymbol{a})$ forms a diagonal matrix based on $\boldsymbol{a}$. The operator $\operatorname{vec}(\boldsymbol{A})$ yields an $R I$ dimensional vector that stacks the $R$ columns of $\boldsymbol{A} \in \mathbb{C}^{I \times R}$ on top of each other. The operator vecdiag $(\boldsymbol{D})$ forms a vector $\boldsymbol{d} \in \mathbb{C}^{R \times 1}$ from the diagonal elements of matrix $\boldsymbol{D} \in \mathbb{C}^{R \times R}$, while $D_{i}(\boldsymbol{A})$ is a diagonal matrix constructed from the $i$-th row of $\boldsymbol{A}$.

Moreover, the Kronecker product and outer product operators are denoted by $\otimes$ and $\circ$, respectively. The KhatriRao product between two matrices $\boldsymbol{A} \in \mathbb{C}^{I \times R}$ and $\boldsymbol{B}=$ $\left[\boldsymbol{b}_{1}, \cdots, \boldsymbol{b}_{R}\right] \in \mathbb{C}^{J \times R}$, denoted by $\diamond$, is their column-wise Kronecker product as below

$$
\boldsymbol{A} \diamond \boldsymbol{B} \doteq[\boldsymbol{A}(:, 1) \otimes \boldsymbol{B}(:, 1), \cdots, \boldsymbol{A}(:, R) \otimes \boldsymbol{B}(:, R)] .
$$

In this paper, the following property of the Khatri-Rao product will be used

$$
\boldsymbol{A} \operatorname{diag}\left(\boldsymbol{x}^{T}\right) \boldsymbol{B}^{T}=\left(\boldsymbol{x}^{\boldsymbol{T}} \diamond \boldsymbol{A}\right) \boldsymbol{B}^{T}
$$

where $\boldsymbol{x}$ is an $R$-dimensional column vector.

\section{SYSTEM MODEL}

Consider a MIMO-OFDM wireless communication system employing $M$ transmit antennas and $K$ receive antennas. In the frequency domain, information transmission is partitioned into groups of $F$ neighboring subcarriers, and spacefrequency coding is applied across these $F$ subcarriers. The transmission time-frame is composed of a collection of $N$ short time-slots of $P$ symbol periods each.

The information symbols stream is first parsed into symbol vectors $\boldsymbol{s}_{n} \in \mathbb{C}^{M \times 1}$. Each symbol vector is linearly precoded across $P$ symbol periods by means of a set of unitary $M \times M$ space-time modulation matrices $\left\{\boldsymbol{G}_{1}, \cdots, \boldsymbol{G}_{P}\right\}$. During the $p$-th symbol period, $\boldsymbol{G}_{p}$ rotates the components of the symbol vector $\boldsymbol{s}_{n}$ and loads a combination of these components into the $M$ transmit antennas. The precoded symbol vector is then used to modulate the $F$ subcarriers through a linear block-coding matrix $\boldsymbol{W} \in \mathbb{C}^{F \times M}$. Along the same lines of [11], we choose $\boldsymbol{W}$ as a Vandermonde matrix with $(f, m)$-th entry given by

$$
[\boldsymbol{W}]_{f, m}=e^{j(f-1)(m-1) \frac{2 \pi}{M}}
$$

In this paper, we focus in the case $F \geq M$.

The above space-frequency coding process can be summarized as

$$
\boldsymbol{C}_{n, p}=\operatorname{diag}\left(\boldsymbol{G}_{p} \boldsymbol{s}_{n}\right) \boldsymbol{W}^{T} \in \mathbb{C}^{M \times F}
$$

where $C_{n, p}$ is the space-frequency code matrix transmitted during the $p$-th symbol period of the $n$-th time-slot. Assuming that the channel is constant over the whole time-frame, the discrete-time baseband equivalent model for the received signal is given by

$$
\boldsymbol{Y}_{n, p}=\sqrt{\frac{\rho}{M}} \boldsymbol{H} \boldsymbol{C}_{n, p}+\boldsymbol{V}_{n, p} \in \mathbb{C}^{K \times F},
$$

where $\boldsymbol{Y}_{n, p}, n=1, \cdots, N, p=1, \cdots, P$ denotes the complex received signal matrix during the $p$-th symbol period of the $n$-th time-slot, and $\boldsymbol{V}_{n, p}$ denotes zero-mean circularly symmetric complex Gaussian (ZMCSCG) noise. The channel matrix $\boldsymbol{H}$ has i.i.d $\mathrm{CN}(0,1)$ entries, and $\rho$ denotes the signalto-noise ratio at each receive antenna.

\subsection{Choice of $\boldsymbol{G}_{p}$}

In consistent with [11], $\boldsymbol{G}_{p}$ has the following form

$$
\boldsymbol{G}_{p}=\boldsymbol{\Theta} \operatorname{diag}\left(\boldsymbol{a}_{p}\right) \in \mathbb{C}^{M \times M}
$$

where $\Theta \in \mathbb{C}^{M \times M}$ is a discrete Fourier transform (DFT) matrix, and $\boldsymbol{a}_{p}=\left[1, e^{j \phi}, \cdots, e^{j(M-1) \phi}\right] \in \mathbb{C}^{1 \times M}$ is a phase rotation vector, with $\phi$ being an elementary rotation that is randomly varied at the transmitter and can be optimized for a given $M$ and modulation type. Note that this model generalizes the model of $[14,15]$ in the sense that it considers $P$ different rotation matrices that are time varying. Our model reduces to that of $[14,15]$ for $P=1$. In $[14,15]$, the elementary rotation $\alpha_{1}$ is assumed to be known. In this paper, we relax this assumption by considering that all $\alpha$ 's are unknown except for $\alpha_{1}$, which is the reference phase whose value is 0 .

\subsection{Receiver Formulation}

The receiver signal (5) can be expressed as

$$
\boldsymbol{Y}_{n, p}=\sqrt{\frac{\rho}{M}} \boldsymbol{H} D_{p}\left(\boldsymbol{A} D_{n}(\boldsymbol{S}) \boldsymbol{\Theta}^{T}\right) \boldsymbol{W}^{T}+\boldsymbol{V}_{n, p}
$$

where $S \in \mathbb{C}^{N \times M}$ is a symbol matrix whose rows are the symbol vectors $\boldsymbol{s}_{n}^{T}, n=1, \cdots, N$, and $\boldsymbol{A} \in \mathbb{C}^{P \times M}$ collects all phase rotation vectors throughout the $P$ symbol periods.

$$
\boldsymbol{A}=\left[\begin{array}{c}
\boldsymbol{a}_{1} \\
\vdots \\
\boldsymbol{a}_{P}
\end{array}\right]=\left[\begin{array}{cccc}
1 & 1 & \cdots & 1 \\
1 & e^{j \phi_{2}} & \cdots & e^{j(M-1) \phi_{2}} \\
\vdots & \vdots & \ddots & \vdots \\
1 & e^{j \phi_{P}} & \cdots & e^{j(M-1) \phi_{P}}
\end{array}\right]
$$

Denoting $\boldsymbol{Y}_{n}=\left[\boldsymbol{Y}_{n, 1}^{T}, \cdots, \boldsymbol{Y}_{n, P}^{T}\right]^{T} \in \mathbb{C}^{P K \times F}$, it follows from the equality in (2) that

$$
\boldsymbol{Y}_{n}=\sqrt{\frac{\rho}{M}}\left[\left(\boldsymbol{A} D_{n}(\boldsymbol{S}) \boldsymbol{\Theta}^{T}\right) \diamond \boldsymbol{H}\right] \boldsymbol{W}^{T}+\boldsymbol{V}_{n}
$$


Similarly, employing $\boldsymbol{Y}=\left[\boldsymbol{Y}_{1}^{T}, \cdots, \boldsymbol{Y}_{N}^{T}\right]^{T} \in \mathbb{C}^{N P K \times F}$ to collect the received data for all $N$ time-slots, yields

$$
\boldsymbol{Y}=\sqrt{\frac{\rho}{M}}\left[\left((\boldsymbol{S} \diamond \boldsymbol{A}) \boldsymbol{\Theta}^{T}\right) \diamond \boldsymbol{H}\right] \boldsymbol{W}^{T}+\boldsymbol{V}
$$

The signal part corresponds to the nested PARAFAC decomposition. In $[8,16]$, the conditions for the essential uniqueness of the PARAFAC decomposition of a tensor, which means it can be determined up to column scaling and permutation, is established. By applying the uniqueness conditions to the problem at hand, the uniqueness of the nested PARAFAC model (10) is guaranteed, if every factor matrix has no pair of proportional columns, and if

$$
\begin{gathered}
\min (N P, M)+\min (K, M)+\min (F, M) \geq 2 M+2 \\
\min (N, M)+\min (P, M) \geq M+2 .
\end{gathered}
$$

\section{ALGORITHM DEVELOPMENT}

To formulate the receiver algorithm, a semi-blind approach is assumed, where only the frequency block-coding matrix $\boldsymbol{W}$ and the fixed constellation rotation matrix $\Theta$ are perfectly known. Before proceeding, we first reformulate the data model in Section 2.2 in tensor form.

\subsection{Tensor formulation}

For notational convenience, let $\boldsymbol{T} \in \mathbb{C}^{N P \times M}$ be the inner PARAFAC part of (10), namely

$$
\boldsymbol{T}=(\boldsymbol{S} \diamond \boldsymbol{A}) \boldsymbol{\Theta}^{T}
$$

In tensor form, (12) can be represented as

$$
\mathcal{T}=\mathcal{I}_{M} \times{ }_{1} S \times{ }_{2} A \times{ }_{3} \Theta \in \mathbb{C}^{N \times P \times M}
$$

where $\mathcal{I}_{M}$ represents the identity tensor of size $M \times M \times M$, and $\times_{r}, r=1, \cdots, R$, is the $r$-mode product operator.

Likewise, (10) can be rewritten in tensor form as

$$
\mathcal{Y}=\sqrt{\frac{\rho}{M}} \cdot \mathcal{I}_{M} \times{ }_{1} \boldsymbol{H} \times{ }_{2} \boldsymbol{W} \times{ }_{3} \boldsymbol{T}+\mathcal{V} \in \mathbb{C}^{K \times F \times N P}
$$

where $\mathcal{V}$ is the noise tensor.

According to the $r$-mode unfolding definition [17], we have $\boldsymbol{T}=[\mathcal{T}]_{3}^{T}$ and $\boldsymbol{Y}=[\mathcal{Y}]_{2}^{T}$.

\subsection{ALS solution}

The ALS algorithm [11] consists in two alternating least square (ALS) based estimation stages. The first stage provides estimates of $\boldsymbol{T}$ and $\boldsymbol{H}$ by fitting the outer PARAFAC model to the received signal $\mathcal{Y}$. The second stage estimate $S$ and $\boldsymbol{A}$ by fitting the inner PARAFAC model to tensor $\mathcal{T}$.

1) First stage
Initialization: Set $i=0$, randomly generate $\hat{\boldsymbol{H}}$.

1a) $i=i+1$

1b) Compute the LS estimate of $\hat{\boldsymbol{T}}$

$$
\left.\hat{\boldsymbol{T}}=[\boldsymbol{Y}]_{3} \cdot\left[(\hat{\boldsymbol{H}} \diamond \boldsymbol{W})^{\dagger}\right)\right]^{T}
$$

1c) Compute the LS estimate of $\hat{\boldsymbol{H}}$

$$
\left.\hat{\boldsymbol{H}}=[\boldsymbol{Y}]_{1} \cdot\left[(\boldsymbol{W} \diamond \hat{\boldsymbol{T}})^{\dagger}\right)\right]^{T}
$$

Repeat $1 a)$ to $1 c$ ) until convergence.

Due to the knowledge of matrices $\boldsymbol{W}$, permutation ambiguity does not exist in the estimated factor matrices of the outer PARAFAC. Besides, the ALS solution [11] assumed that at the beginning of the data transmission, a known frame head of $M$ symbols is transmitted, so that the first row of $\boldsymbol{S}$ is known. This together with the fact that the first row of $\boldsymbol{A}$ is also known (it is equal to the all 1's vector) allows us to know the first row in matrix $\boldsymbol{T}$ according to (12). Then the scaling ambiguity from the estimates $\hat{\boldsymbol{T}}$ and hence from $\hat{\boldsymbol{H}}$ can be removed.

2) Second stage

Initialization: Reconstruct vector $\mathcal{T}$ from $\hat{\boldsymbol{T}}$. Set $i=0$, randomly generate $\hat{A}$.

2a) $i=i+1$

2b) Compute the LS estimate of $\hat{S}$

$$
\left.\hat{\boldsymbol{S}}=[\boldsymbol{T}]_{1} \cdot\left[(\hat{\boldsymbol{A}} \diamond \boldsymbol{\Theta})^{\dagger}\right)\right]^{T}
$$

2c) Compute the LS estimate of $\hat{\boldsymbol{A}}$

$$
\left.\hat{\boldsymbol{A}}=[\boldsymbol{\mathcal { T }}]_{2} \cdot\left[(\boldsymbol{\Theta} \diamond \hat{\boldsymbol{S}})^{\dagger}\right)\right]^{T}
$$

Repeat $2 a$ ) to $2 c$ ) until convergence.

Likewise, since $\Theta$ is known, permutation ambiguity does not exist in the estimated factor matrices of the inner PARAFAC. And since the first rows of both $\boldsymbol{S}$ and $\boldsymbol{A}$ are known, the scaling ambiguity of $\hat{\boldsymbol{S}}$ and $\hat{\boldsymbol{A}}$ can be readily removed as well.

\subsection{Proposed LS-KRF solution}

Now we propose a closed form solution via least squares Khatri-Rao factorization (LS-KRF) for blind joint detection and channel estimation. First we estimate $\boldsymbol{T}$ and $\boldsymbol{H}$ via the outer PARAFAC model. The 2-mode unfolding of tensor $\mathcal{Y}$ gives

$$
[\mathcal{Y}]_{2}=\boldsymbol{W} \cdot[(\boldsymbol{T} \diamond \boldsymbol{H})]^{T} .
$$

After multiplying both sides in (19) by the pseudo-inverse of $W$ and then taking the transpose, we get

$$
(\boldsymbol{T} \diamond \boldsymbol{H})=\left(\boldsymbol{W}^{\dagger} \cdot[\boldsymbol{Y}]_{2}\right)^{T} \in \mathbb{C}^{N P K \times M}
$$

The unvectorization of the $m$ th column of $(\boldsymbol{T} \diamond \boldsymbol{H}), m=$ $1, \cdots, M$, yields a matrix $\boldsymbol{Q}_{m} \in \mathbb{C}^{N P \times K}$. According to (1), in the presence of noise, $\boldsymbol{Q}_{m}$ is a rank-one matrix

$$
\boldsymbol{Q}_{m}=\boldsymbol{H}(:, m) \circ \boldsymbol{T}_{0}(:, m) .
$$


Hence we can apply SVD to $\boldsymbol{Q}_{m}$ to obtain the estimate $\boldsymbol{H}$ (: $, m) \in \mathbb{C}^{N P \times 1}$ and $\boldsymbol{T}(:, m) \in \mathbb{C}^{K \times 1}$. Suppose after SVD, we have

$$
\boldsymbol{Q}_{m}=\boldsymbol{U}_{m} \cdot \Sigma_{m} \cdot \boldsymbol{V}_{m}^{H},
$$

then the estimates of $\boldsymbol{H}(:, m)$ and $\boldsymbol{T}(:, m)$ are given by

$$
\hat{\boldsymbol{T}}(:, m)=\sqrt{\sigma_{1 m}} \boldsymbol{U}_{m}(:, 1) \in \mathbb{C}^{N P \times 1}
$$

and

$$
\hat{\boldsymbol{H}}(:, m)=\sqrt{\sigma_{1 m}} \boldsymbol{V}_{m}^{*}(:, 1) \in \mathbb{C}^{K \times 1} .
$$

Similar with the ALS solution, the scaling ambiguity from $\hat{T}$ can be removed by knowing that the first row in $T$ is known.

With the new estimated $\hat{\boldsymbol{T}}$, we can apply the LS-KRF solution to the inner PARAFAC model in a similar way. By multiplying both sides in (12) by $\Theta$, we get

$$
(\hat{\boldsymbol{S}} \diamond \hat{\boldsymbol{A}})=\hat{\boldsymbol{T}} \cdot \boldsymbol{\Theta} \in \mathbb{C}^{N P \times M}
$$

After unvectorizing the $m$ th column of $\hat{\boldsymbol{S}} \diamond \hat{\boldsymbol{A}}$ into a rank-1 matrix $\boldsymbol{Q}_{m}^{\prime} \in \mathbb{C}^{N \times P}, m=1, \cdots, M$, the $\mathrm{SVD}$ an be exploited to obtain the dominant vectors $\boldsymbol{u}_{1 m}^{\prime} \in \mathbb{C}^{N \times 1}, \boldsymbol{v}^{\prime}{ }_{1 m} \in \mathbb{C}^{P \times 1}$ and the dominant singular value $\sigma_{1 m}^{\prime}$. In doing so we will find that

$$
\begin{aligned}
& \hat{\boldsymbol{S}}=\left[\begin{array}{lll}
\sqrt{\sigma_{11}^{\prime}} \boldsymbol{u}_{11}^{\prime} & \cdots & \sqrt{\sigma_{1 M}^{\prime}} \boldsymbol{u}_{1 M}^{\prime}
\end{array}\right] \in \mathbb{C}^{N \times M} \\
& \hat{\boldsymbol{A}}=\left[\begin{array}{llll}
\sqrt{\sigma_{11}^{\prime}} \boldsymbol{v}_{11}^{\prime} & \cdots & \sqrt{\sigma_{1 M}^{\prime}} \boldsymbol{v}_{1 M}^{\prime}
\end{array}\right]^{*} \in \mathbb{C}^{P \times M}
\end{aligned}
$$

Again, the scaling ambiguity in $\hat{\boldsymbol{S}}$ and $\hat{\boldsymbol{A}}$ can be readily removed in a similar way with that in the ALS solution.

\section{NUMERICAL EXAMPLES}

We present simulation results demonstrating the performance of the proposed LS-KRF semi-blind receiver. More specifically, the bit error rate (BER), the normalized mean square error (NMSE) of the estimated channel and the mean processing time will be considered for performance evaluation. Each result represents an average of 10000 independent Monte Carlo runs. At each run, the transmitted symbols are drawn from a QPSK sequence, and the channel coefficients are i.i.d ZMCSCG distributed with equal power. The additive noise power is scaled according to the signal-to-noise ratio (SNR), which is defined as SNR $=\left\|\boldsymbol{Y}_{0}\right\|_{F}^{2} /\|\boldsymbol{V}\|_{F}^{2}$. The NMSE of the estimated channel is defined as

$$
\mathrm{NMSE}=\|\hat{\boldsymbol{H}}-\boldsymbol{H}\|_{F}^{2} /\|\boldsymbol{H}\|_{F}^{2} .
$$

where $\|\cdot\|_{F}$ denotes the Frobenius norm of a matrix, which is defined as the square root of the sum of the absolute squares of its elements. Since different parameter settings result in similar results, herein we consider only one configuration, where $K=2, M=4, F=5, N=5, P=3$.
First, we evaluate the BER performance of the proposed LS-KRF receiver. In Figure 1, we evaluate its BER performance and compare with that of the ALS solution at different number of iterations $k_{i}$, where SNR is set as $0 \mathrm{~dB}$. It can be seen that when the number of iterations in the latter is sufficiently large, its BER will converge to a stationary point that is equal to that of LS-KRF. However, when the number of iterations is below the threshold number required $\left(k_{T}=7\right)$ to reach the stationary point, the BER obtained with the ALS receiver is not stable, and presents substantial fluctuations, with an average larger than that of the LS-KRF scheme. This behavior could be explained by the procedure of the ALS receiver, which provides a gradual improvement on the estimates at each iteration. In contrast, the closed-form LS-KRF receiver has a constant performance that is not dependent on the number of iterations.

Figure 2 plots the normalized mean square error (NMSE) of the channel estimate for different number of iterations at $\mathrm{SNR}=0 \mathrm{~dB}$. Again, the NMSE of ALS method depends on the number of iterations. Its NMSE converges to the same value as that of LS-KRF algorithm for sufficiently large number of iterations, and experiences variation with average worse performance when the number of iterations is relatively small.

Figure 3 shows the mean processing time in seconds in ALS receiver as a function of the actual number of iterations. Here, the number of iterations in the outer and inner PARAFAC are set the same value. It can be seen that the mean processing time of ALS increases linearly with the number of iterations, and when $k_{i} \geq 5$ it starts to exceed the processing time needed in LS-KRF. To achieve the same BER and NMSE performance as that in LS-KRF, the minimum number of iterations required is $k_{T}=7>5$ and hence more processing time is required in ALS.

Similar observations are obtained for other SNRs. In Figures 4 and 5, the LS-KRF and ALS receivers are compared in terms of BER and NMSE for various SNRs. In the ALS scheme, the outer iteration stops when the root square reconstruction change (RSRC) between two successive iterations given by

$$
\operatorname{RSRC}_{\text {out }}(k)=\sqrt{\frac{\left\|\hat{\mathcal{Y}}_{k, 0}-\hat{\mathcal{Y}}_{k-1,0}\right\|_{\mathrm{F}}^{2}}{K \times F \times N P}}
$$

is smaller than the predefined threshold $\eta_{1}$, where $\|\cdot\|_{\mathrm{F}}$ denotes the Frobenius norm of a tensor, which is defined as the square root of the sum of the absolute squares of its elements. In a similar manner, the inner iteration stops when the RSRC between two successive iterations given by

$$
\operatorname{RSRC}_{\text {in }}(k)=\sqrt{\frac{\left\|\hat{\mathcal{T}}_{k, 0}-\hat{\mathcal{T}}_{k-1,0}\right\|_{\mathrm{F}}^{2}}{N \times P \times M}}
$$

is smaller than the predefined threshold $\eta_{2}$. As shown in Figures 4 and 5, the LS-KRF and ALS receivers give very close 
performance in terms of BER and NMSE, when the convergence thresholds for the two iterations in the latter are set as $\eta_{1}=\eta_{2}=10^{-4}$. In Figure 6 top, the corresponding mean processing time in seconds for different SNRs are compared between the ALS and LS-KRF receiver. Note that the mean processing time required in the latter is constant with SNR since it is non-iterative, while that required in the former decreases with SNR since it is an iterative receiver where the number of iterations required for both the outer and inner PARAFAC decreases with SNR, as shown in Figure 6 bottom. Note that the closed-form LS-KRF receiver requires less processing time in low-to-medium SNR regimes.

\section{CONCLUSION}

In this paper, we have proposed a low-complexity semi-blind receiver based on the least squares Khatri-Rao factorization (LS-KRF) for the blind joint channel and symbol estimation of MIMO-OFDM systems with space-time-frequency diversity coding. The proposed LS-KRF receiver is a closed-form solution that provides performance stability as well as lower computational complexity compared to the ALS based receiver in low-to-medium SNR range. Therefore, the proposed receiver algorithm is more attractive in situations where computational complexity and processing delay are limited.

\section{REFERENCES}

[1] G. L. Stuber, J. R. Barry, S. W. McLaughlin, Ye Li, M. A. Ingram, and T. G. Pratt, "Broadband MIMOOFDM wireless communications," Proc. IEEE, vol. 92, no. 2, pp. 271-294, 2004.

[2] S. M. Alamouti, "A simple transmit diversity technique for wireless communications," IEEE J. Sel. Areas Commun., vol. 16, no. 8, pp. 1451-1458, 1998.

[3] D. Agrawal, V. Tarokh, A. Naguib, and N. Seshadri, "Space-time coded OFDM for high data-rate wireless communication over wideband channels," in Proc. 48th IEEE Vehicular Technology Conf. VTC 98, Ottawa, Canada, May 1998, vol. 3, pp. 2232-2236.

[4] V. Tarokh, H. Jafarkhani, and A. R. Calderbank, "Spacetime block codes from orthogonal designs," IEEE Trans. Inf. Theory, vol. 45, no. 5, pp. 1456-1467, July 1999.

[5] H. Bolcskei and A. J. Paulraj, "Space-frequency coded broadband ofdm systems," in Proc. WCNC Wireless Communications and Networking Confernce 2000 IEEE, Chicago, Illinois, USA, September 2000, vol. 1, pp. 1-6.

[6] W. Su, Z. Safar, and K. J. R. Liu, "Full-rate fulldiversity space-frequency codes with optimum coding advantage," IEEE Trans. Inf. Theory, vol. 51, no. 1, pp. 229-249, 2005.

[7] Lei Shao and S. Roy, "Rate-one space-frequency block codes with maximum diversity for mimo-ofdm," IEEE Trans. Wireless Commun., vol. 4, no. 4, pp. 1674-1687, 2005.

[8] N. D. Sidiropoulos and R. S. Budampati, "Khatri-Rao space-time codes," IEEE Trans. Signal Process., vol. 50, no. 10, pp. 2396-2407, 2002.

[9] A. L. F. de Almeida, G. Favier, and J. C. M. Mota, "Space-time multiplexing codes: A tensor modeling approach," in Proc. IEEE 7th Workshop Signal Processing Advances in Wireless Communications SPAWC '06, Cannes, France, July 2006, pp. 1-5.

[10] A. L. F. de Almeida, G. Favier, and J. C. M. Mota, "A constrained factor decomposition with application to MIMO antenna systems," IEEE Trans. Signal Process., vol. 56, no. 6, pp. 2429-2442, 2008.

[11] A. L. F. de Almeida, "Blind joint detection and channel estimation in space-frequency diversity systems using time-varying linear constellation precoding," in Brazilian Telecommunications Symposium (SBrT'11), Caryatid, Paraná, Brazil, October 2011.

[12] F. Roemer and M. Haardt, "Tensor-based channel estimation and iterative refinements for two-way relaying with multiple antennas and spatial reuse," IEEE Trans. Signal Process., vol. 58, no. 11, pp. 5720-5735, 2010.

[13] J. P. C. L. da Costa, F. Roemer, M. Weis, and M. Haardt, "Robust $R$-D parameter estimation via closed-form PARAFAC," in Proc. Int Smart Antennas (WSA) ITG Workshop, Bremen, Germany, February 2010, pp. 99106.

[14] Yan Xin, Zhengdao Wang, and G. B. Giannakis, "Spacetime diversity systems based on linear constellation precoding," IEEE Trans. Wireless Commun., vol. 2, no. 2, pp. 294-309, 2003.

[15] R. Vishwanath and M. R. Bhatnagar, "Optimum linear constellation precoding for space time wireless systems," Wirel. Pers. Commun., vol. 40, No. 4, pp. 511521, Mar. 2007.

[16] J. B. Kruskal, "Three-way arrays: rank and uniqueness of trilinear decompositions, with application to arithmetic complexity and statistics," Linear Algebra Application, vol. 18, pp. 95138, 1977.

[17] L. de Lathauwer, B. de Moor, and J. Vanderwalle, "A multilinear singular value decomposition," SIAM J. Matrix Anal. Appl., vol. vol. 21, no. 4, pp. 1253-1278, 2000. 


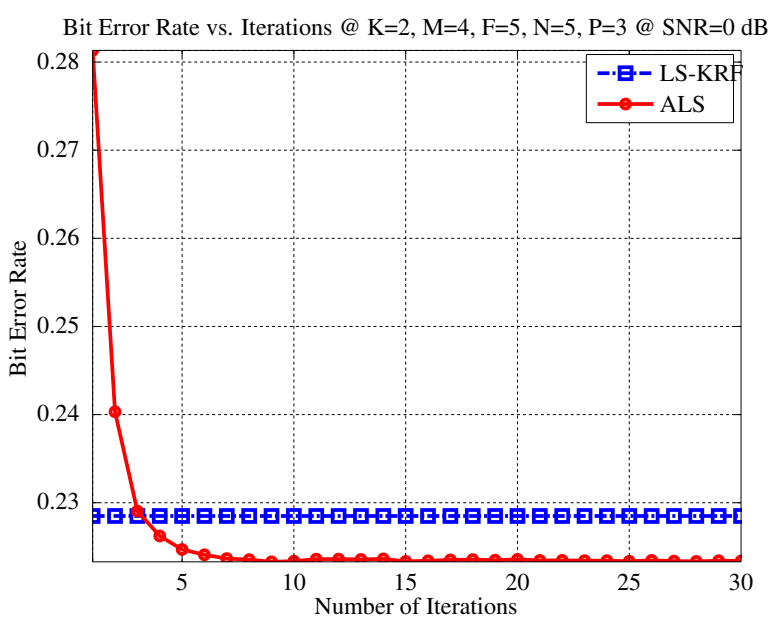

Fig. 1: BER versus number of iterations.

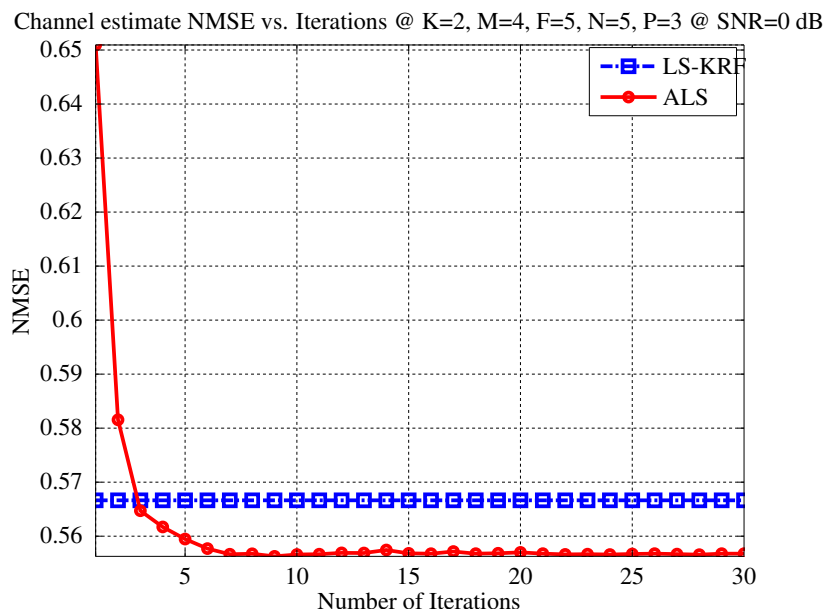

Fig. 2: Channel estimate NMSE versus number of iterations.

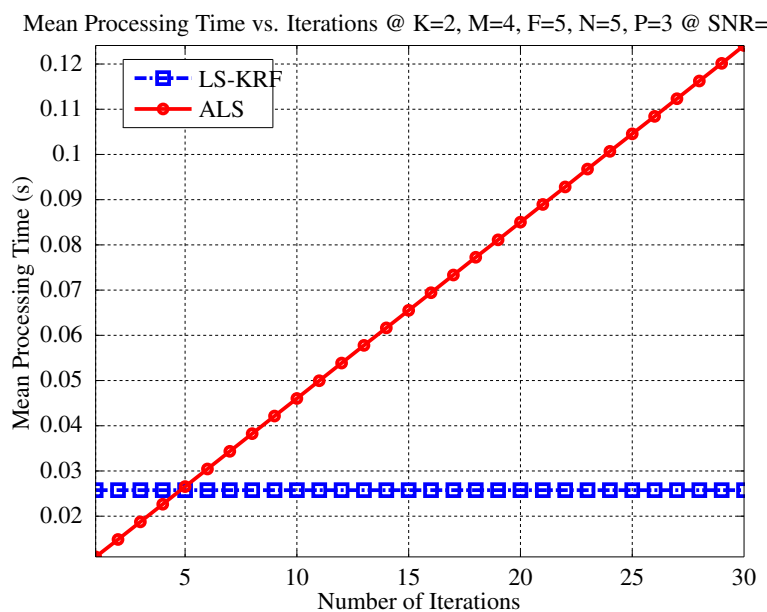

Fig. 3: Mean processing time versus number of iterations.

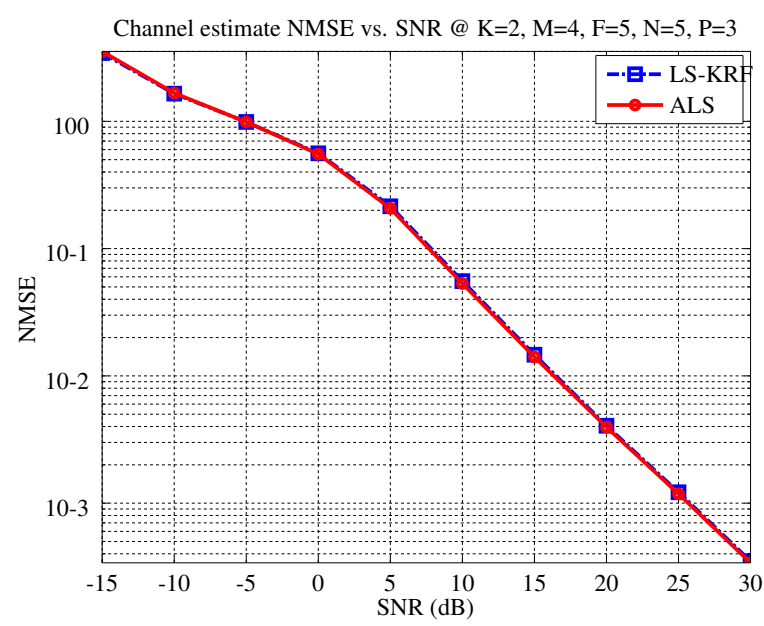

Fig. 4: BER versus SNR of the semi-blind decoding.

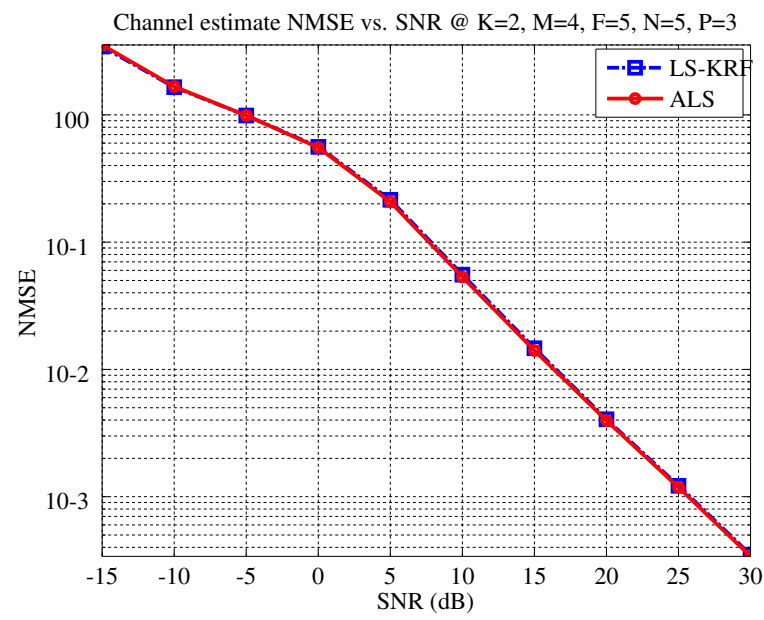

Fig. 5: Channel estimate NMSE versus SNR.

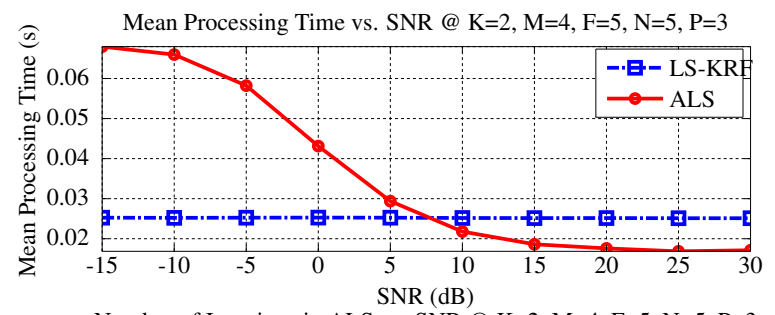

Number of Iterations in ALS vs. SNR @ K=2, M=4, F=5, N=5, P=3

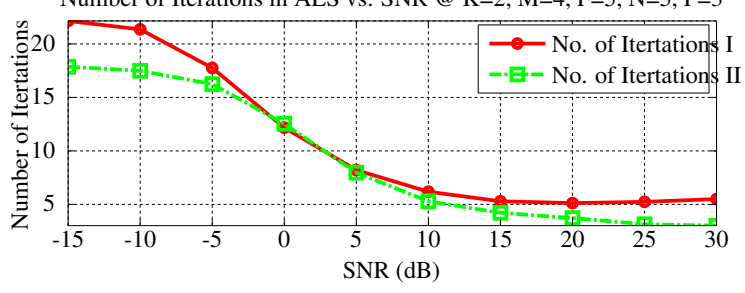

Fig. 6: Mean Processing Time versus SNR. 